\title{
Multiscale lattice Boltzmann approach to modeling gas flows
}

\author{
Jianping Meng* and Yonghao Zhang ${ }^{\dagger}$ \\ Department of Mechanical Engineering, University of Strathclyde, Glasgow, G1 1 XJ, UK \\ Xiaowen Shan \\ Exa Corporation, 55 Network Drive, Burlington, Massachusetts 01803, USA
}

(Dated: December 20, 2010)

\begin{abstract}
For multiscale gas flows, kinetic-continuum hybrid method is usually used to balance the computational accuracy and efficiency. However, the kinetic-continuum coupling is not straightforward since the coupled methods are based on different theoretical frameworks. In particular, it is not easy to recover the non-equilibrium information required by the kinetic method which is lost by the continuum model at the coupling interface. Therefore, we present a multiscale lattice Boltzmann (LB) method which deploys high-order LB models in highly rarefied flow regions and low-order ones in less rarefied regions. Since this multiscale approach is based on the same theoretical framework, the coupling precess becomes simple. The non-equilibrium information will not be lost at the interface as low-order LB models can also retain this information. The simulation results confirm that the present method can achieve modeling accuracy with reduced computational cost.
\end{abstract}

PACS numbers: 47.11.-j, 47.61.-k

\section{INTRODUCTION}

Many engineering problems involve multi-scale gas flows, e.g. gas flows in micro/nano-fluidic devices [1]. Since the flow regions can be highly rarefied (nonequilibrium), the conventional continuum theory becomes inappropriate. The rarefaction order of gas flows can be classified by the non-dimensional Knudsen number, Kn, defined as the ratio of the mean free path and the device characteristic length scale. The Navier Stokes equations with no-velocity-slip wall boundary condition are only appropriate in the hydrodynamic regime where $K n<0.001$. In the slip flow regime $(0.001<K n<0.1)$, or the transition flow regime $(0.1<K n<10)$, it is necessary to use kinetic methods, e.g. direct simulation Monte Carlo method (DSMC) method, to describe gas flows. Although the kinetic methods including DSMC are able to simulate flow in the continuum or near-continuum regimes, the computational cost is often very expensive especially for low speed flows[2]. Therefore, kineticcontinuum hybrid methods are naturally employed to deal with mixed flow regimes in typical microfluidic devices operating with a range of Knudsen numbers in different parts, i.e., numerically efficient continuum approach will be employed for the continuum regimes and kinetic approach for the rarefied regimes(see Refs. [3-14] and references therein). The two models are coupled by exchanging information over the "hand-shaking" region or across an interface.

However, the kinetic-continuum coupling is not straightforward since two types of methods are based

\footnotetext{
* Email: jianping.meng@strath.ac.uk

$\dagger$ Author to whom correspondence should be addressed.

Email: yonghao.zhang@strath.ac.uk

‡ Email: xiaowen@exa.com
}

on different theoretical frameworks. While information transferring from the kinetic model to the continuum model is usually a well-defined process, the reverse process is more problematic [13]. It is difficult to recover non-equilibrium information lost by the continuum solvers which is required by the kinetic method. $\mathrm{Al}-$ though the kinetic model can provide necessary information for the continuum model, it can be computationally expensive [5]. The statistical noise associated with the particle methods may also affect the accuracy and stability of the hybrid solver [13]. To effectively model mixed-Kn flows, we introduce a multiscale lattice Boltzmann (LB) method to utilize various order LB models. Since this multiscale method is based on a same theoretical framework, it has distinguished advantages, which has also been demonstrated recently by the unified gaskinetic scheme[15].

The LB method has been proven to be able to simulate hydrodynamic flows with only minimal number of discrete velocities (e.g., nine discrete velocities for a twodimensional problem) [16-20]. For continuum problems, its applicability is ensured by the Chapman-Enskog expansion. Due to its kinetic nature, the LB model has its advantages over other continuum computational methods, including easy implementation of multi-physical mechanisms and the boundary conditions for fluid/wall interactions [17, 28].

The LB method may also offer a flexible framework for rarefied flows, which has recently been demonstrated extensively (see Refs.[19, 21-32] and references therein). It was shown that the key to capturing the rarefaction effects is to choose appropriate discrete velocity sets. Generally, a high-order LB model with larger discrete velocity set describes non-equilibrium effects better [29$31,33]$. In particular, high-order models with modest discrete velocity sets can already accurately capture nonequilibrium effects in rarefied flows over a range of Knud- 
sen numbers [29-31, 33].

Since the LB method offers a solution for simulating gas flows ranging from continuum to rarefied, we can introduce a multiscale method to couple models based on the same LB framework. This can be accomplished by employing higher-order LB models for non-equilibrium flow regions and lower-order LB models for hydrodynamic flow regions. Since the coupled LB models only differ in the chosen discrete velocities without loss of kinetic information at the coupling interface, the information exchange process can be simplified. In particular, non-equilibrium information can be retained in lowerorder LB models, which resolves an obstacle associated with kinetic-continuum hybrid methods. Meanwhile, the LB model can still reserve some advantages of particle method while eliminating the statistical noise.

\section{LATTICE BOLTZMANN METHOD}

\section{A. Lattice Boltzmann equation}

Historically, the LB method was developed from the lattice gas cellular automata. The purpose was to mimic the Navier-Stokes dynamics. However, it was revealed that its applicability should not be limited to the hydrodynamic level [28, 29, 33-39]. There are different theoretical frameworks for LB models e.g., entropic LB models [38-42]. Here, to demonstrate multiscale methodology, we will adopt commonly-used LB models based on the Hermite expansion detailed in Refs.[28, 34-37, 43]. However, the proposed coupling approach can be equally applied to different LB models.

The original BGK equation is given as:

$$
\frac{\partial f}{\partial t}+\boldsymbol{\xi} \cdot \nabla f+\boldsymbol{g} \cdot \nabla_{\xi} f=-\frac{p}{\mu}\left(f-f^{e q}\right),
$$

where $f$ denotes the distribution function, $\boldsymbol{\xi}$ the phase velocity, $p$ the pressure, $\boldsymbol{g}$ the body force and $\mu$ the gas viscosity. Using the well-known Chapman-Enskog expansion, the collision frequency can be represented by the ratio of pressure and gas viscosity, which is convenient to obtain the Knudsen number definition consistent with that of hydrodynamic models. Without losing generality, one can define the following non-dimensional variables:

$$
\begin{gathered}
\hat{\boldsymbol{r}}=\frac{\boldsymbol{r}}{l_{0}}, \hat{\boldsymbol{u}}=\frac{\boldsymbol{u}}{\sqrt{R T_{0}}}, \hat{t}=\frac{\sqrt{R T_{0}} t}{l_{0}}, \\
\hat{\boldsymbol{g}}=\frac{l_{0} \boldsymbol{g}}{R T_{0}}, \hat{\boldsymbol{\xi}}=\frac{\boldsymbol{\xi}}{\sqrt{R T_{0}}}, \hat{T}=\frac{T}{T_{0}},
\end{gathered}
$$

where $\boldsymbol{u}$ is the macroscopic velocity, $R$ the gas constant, $T$ the gas temperature, $T_{0}$ the reference temperature, $r$ the spatial position and $l_{0}$ the characteristic length of the flow system. The symbol hat, which denotes a dimensionless value, will hereinafter be omitted. The Knudsen number can be defined by using macroscopic properties as below:

$$
K n=\frac{\mu \sqrt{R T_{0}}}{p l_{0}}
$$

Based on these non-dimensional variables, the nondimensional form of the BGK equation becomes

$$
\frac{\partial f}{\partial t}+\boldsymbol{\xi} \cdot \nabla f+\boldsymbol{g} \cdot \nabla_{\xi} f=-\frac{1}{K n}\left(f-f^{e q}\right),
$$

where the Maxwell distribution in $D$-dimensional Cartesian coordinates can be written as

$$
f^{e q}=\frac{\rho}{(2 \pi T)^{D / 2}} \exp \left[\frac{-(\boldsymbol{\xi}-\boldsymbol{u})^{2}}{2 T}\right] .
$$

For solving Eq.(4), the velocity space can be firstly discretized by projecting the distribution function onto a functional space spanned by the orthogonal Hermite basis $[28,44]$ :

$$
f(\boldsymbol{r}, \boldsymbol{\xi}, t) \approx f^{N}(\boldsymbol{r}, \boldsymbol{\xi}, t)=\omega(\boldsymbol{\xi}) \sum_{n=0}^{N} \frac{1}{n !} \boldsymbol{a}^{(n)}(\boldsymbol{r}, t) \boldsymbol{\chi}^{(n)}(\boldsymbol{\xi})
$$

where $\chi^{(n)}$ is the $n$th order Hermite polynomial, and $\omega(\boldsymbol{\xi})$ is the weight function, which are given by

$$
\begin{gathered}
\chi^{(n)}(\boldsymbol{\xi})=\frac{(-1)^{n}}{\omega(\boldsymbol{\xi})} \nabla^{n} \omega(\boldsymbol{\xi}), \\
\omega(\boldsymbol{\xi})=\frac{1}{(2 \pi)^{D / 2}} \mathrm{e}^{-\xi^{2} / 2} .
\end{gathered}
$$

The coefficients $\boldsymbol{a}^{(n)}$ are

$$
\begin{aligned}
\boldsymbol{a}^{(n)} & =\int f \boldsymbol{\chi}^{(n)} d \boldsymbol{\xi} \approx \int f^{(N)} \chi^{(n)} d \boldsymbol{\xi} \\
& =\sum_{\alpha=1}^{d} \frac{w_{\alpha}}{\omega\left(\boldsymbol{\xi}_{\alpha}\right)} f^{(N)}\left(\boldsymbol{r}, \boldsymbol{\xi}_{\alpha}, t\right) \boldsymbol{\chi}^{(n)}\left(\boldsymbol{\xi}_{\alpha}\right) .
\end{aligned}
$$

The equilibrium distribution should also be expanded as $[28]$

$$
f^{e q} \approx \omega(\boldsymbol{\xi}) \sum_{n=0}^{N} \frac{1}{n !} \boldsymbol{a}_{e q}^{(n)} \chi^{(n)}(\boldsymbol{\xi})
$$

where the coefficient $a_{e q}^{(n)}$ for the equilibrium distribution is

$$
\boldsymbol{a}_{e q}^{(n)}=\int f^{e q} \boldsymbol{\chi}^{(n)} d \boldsymbol{\xi}
$$

$w_{\alpha}$ and $\boldsymbol{\xi}_{\alpha}, a=1, \cdots, d$, are the weights and abscissae of a Gauss-Hermite quadrature of degree $\geq 2 N$ respectively. Therefore, the Maxwell distribution is approximated by up to $N$ Hermite polynomials. The body force term $F(\boldsymbol{r}, \boldsymbol{\xi}, t)=\boldsymbol{g} \cdot \nabla_{\xi} f$ can also be approximated as $[28,37]$ 


$$
F(\boldsymbol{r}, \boldsymbol{\xi}, t)=\omega \sum_{n=1}^{N} \frac{1}{(n-1) !} \boldsymbol{g} \boldsymbol{a}^{(n-1)} \boldsymbol{\chi}^{(n)} .
$$

It was shown that Eq.(4) with the first-order Hermite expansion is sufficient to capture the rarefaction effects for isothermal and incompressible flows[33]. On the other hand, the second order expansion has been proven to be able to model various Navier-Stokes level problems $[17,19]$. Therefore, the second order approximation of the equilibrium distribution and the body force will be used hereinafter, as given below:

$$
f^{e q} \approx \omega(\boldsymbol{\xi}) \rho\left\{1+\boldsymbol{\xi} \cdot \boldsymbol{u}+\frac{1}{2}\left[(\boldsymbol{\xi} \cdot \boldsymbol{u})^{2}-u^{2}+(T-1)\left(\xi^{2}-D\right)\right]\right\},
$$

$$
F(\boldsymbol{r}, \boldsymbol{\xi}, t) \approx \omega(\boldsymbol{\xi}) \rho\{\boldsymbol{g} \cdot \boldsymbol{\xi}+(\boldsymbol{g} \cdot \boldsymbol{\xi})(\boldsymbol{u} \cdot \boldsymbol{\xi})-\boldsymbol{g} \cdot \boldsymbol{u}\},
$$

where $T$ should be unity for isothermal problems and $\rho$ is constant for incompressible problems. Since we will only demonstrate the capability of our multiscale scheme for isothermal problems here, the terms related to temperature will be omitted for convenience.

The discrete velocity set is revealed to be of upmost importance in determining model accuracy for rarefaction effects [33]. For the Navier-Stokes level problems, several sets have been found to be applicable, e.g., the well known D2Q9[45] model for two-dimensional flows. To capture higher-order rarefaction effects, more discrete velocities are required. Some modest discrete velocity sets were shown (e.g., D2Q16 and D2Q36) to be able to capture non-equilibrium effects for flows over a broad range of Knudsen numbers[29-31, 33]. Nevertheless, highly accurate discrete velocity set is required for the flows with large Knudsen number. Therefore, coupling high-order and low-order LB models can save computational costs without sacrificing simulation accuracy for gas flows with mixed Knudsen numbers.

Discrete velocity sets can be obtained from several ways, see Refs.[28, 39, 40, 43]. A direct method is utilizing the roots of Hermite polynomials[28]. In onedimension, the discrete velocities $\xi_{\alpha}$ are just the roots of Hermite polynomials, and its corresponding weights are determined by:

$$
w_{\alpha}=\frac{n !}{\left[n \chi^{n-1}\left(\xi_{\alpha}\right)\right]^{2}} .
$$

For higher dimensions, the discrete velocity set can be constructed by using the "production" formulae [28].

Once the discrete velocity set is chosen, Eq.(4) can be discretized as

$$
\frac{\partial f_{\alpha}}{\partial t}+\boldsymbol{\xi}_{\alpha} \cdot \nabla f_{\alpha}=-\frac{1}{K n}\left(f_{\alpha}-f_{\alpha}^{e q}\right)+g_{\alpha},
$$

where $f_{\alpha}=\frac{w_{\alpha} f\left(\boldsymbol{r}, \boldsymbol{\xi}_{\alpha}, t\right)}{\omega\left(\boldsymbol{\xi}_{\alpha}\right)}, f_{\alpha}^{e q}=\frac{w_{\alpha} f^{e q}\left(\boldsymbol{r}, \boldsymbol{\xi}_{\alpha}, t\right)}{\omega\left(\boldsymbol{\xi}_{\alpha}\right)}$ and $g_{\alpha}=$ $\frac{w_{\alpha} F\left(\boldsymbol{r}, \boldsymbol{\xi}_{\alpha}, t\right)}{\omega\left(\boldsymbol{\xi}_{\alpha}\right)}$. Therefore, the LB equation, i.e. Eq.(16), is now obtained by discretizing Eq.(4) in the velocity space.

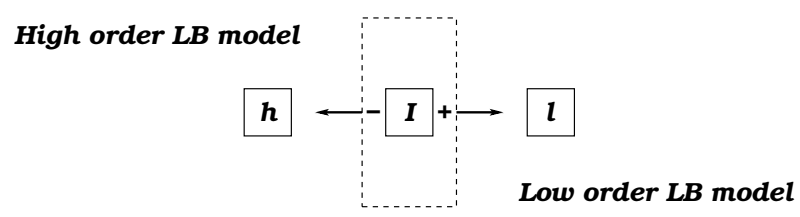

FIG. 1. Schematic illustration of interface treatment where $I$ is the grid on the interface while $h$ and $l$ represent the adjacent grids at the computational domains for high-order and low-order LB models respectively.

\section{B. Coupling scheme}

The key to success of a coupling scheme is appropriate bi-directional extraction and transfer of information at the interface or "hand-shaking"region. Since only LB models are used here, the extraction and transfer of information is in principle seamless. Lower order LB models, in their applicable capacity, can also retain nonequilibrium information which is required by the higherorder models. For instance, the D2Q16 model can already perform well for a range of Knudsen numbers [2931, 33]. The D2Q9 model, which has been used for hydrodynamic simulations, may also capture some nonequilibrium effects $[29,31]$. This is very different from the continuum methods in kinetic-continuum hybrid models where non-equilibrium information is lost.

To correctly transfer information across the interface between two LB models with different discrete velocities, the interface can be treated as a "virtual boundary". Since a properly determined interface should be located at smooth regimes where lower-order models are valid, the relevant information can be obtained by using extrapolation and interpolation techniques. Firstly, the related macroscopic quantities can be calculated by interpolation, so that the equilibrium part of "boundary conditions" is able to be obtained by the Maxwell-Boltzmann distribution. For the non-equilibrium part of information, recall that not only higher-order but also lowerorder LB models can produce accurate non-equilibrium information in the interface flow region. Moreover, the information provided by two models should be completely same on the "boundary". Therefore, the nonequilibrium part of information on the "boundary" for the low-order and high-order LB models can be obtained via extrapolating information on the grids adjacent to the "boundary". It is interesting to note that similar techniques have been used to construct the no-slip boundary condition for continuum problems [46-48].

To illustrate the scheme clearly, it is convenient to discuss in details for a one-dimensional example. However, the same methodology can be generalized for multidimensional problems. The distribution function can be decomposed into its equilibrium $\left(f_{\alpha}^{e q}(I, t)\right)$ and non- 
equilibrium $\left(f_{\alpha}^{n e q}(I, t)\right)$ parts, i.e.,

$$
f_{\alpha}(I, t)=f_{\alpha}^{e q}(I, t)+f_{\alpha}^{n e}(I, t),
$$

where the letter I denotes an interface grid (see Fig.1). The velocity direction needs to be further classified since different discrete velocity sets are used across the interface. Hereinafter, the symbol + denotes the discrete velocities (see Fig.1) pointing to the lower-order LB model side, - to the higher-order LB model side. Firstly, the macroscopic quantities related to the equilibrium distribution can be obtained simply by the linear interpolation, i.e.,

$$
\begin{aligned}
\rho_{I} & =\frac{\rho_{l}+\rho_{h}}{2}, \\
\boldsymbol{u}_{I} & =\frac{\boldsymbol{u}_{l}+\boldsymbol{u}_{h}}{2},
\end{aligned}
$$

where $l$ and $h$ are the interface neighboring grids (see Fig.1). With these quantities, the equilibrium distribution can be written as

$$
\begin{aligned}
& f_{\alpha+}^{e q}(I, t) \approx w_{\alpha+} \rho_{I}\left\{1+\boldsymbol{\xi}_{\alpha+} \cdot \boldsymbol{u}_{I}+\frac{1}{2}\left[\left(\boldsymbol{\xi}_{\alpha+} \cdot \boldsymbol{u}_{I}\right)^{2}-u_{I}^{2}\right]\right\} \\
& f_{\alpha-}^{e q}(I, t) \approx w_{\alpha-} \rho_{I}\left\{1+\boldsymbol{\xi}_{\alpha-} \cdot \boldsymbol{u}_{I}+\frac{1}{2}\left[\left(\boldsymbol{\xi}_{\alpha-} \cdot \boldsymbol{u}_{I}\right)^{2}-u_{I}^{2}\right]\right\}
\end{aligned}
$$

Note, $\boldsymbol{\xi}_{\alpha-}, \boldsymbol{\xi}_{\alpha+}$ and $w_{\alpha-}, w_{\alpha+}$ belong to two different discrete velocity sets. Based on the equilibrium distribution functions, the required information can be transferred cross the interface. Meanwhile, a first order extrapolation scheme is employed to supplement the information for the non-equilibrium part, i.e.,

$$
\begin{gathered}
f_{\alpha+}^{n e q}(I, t)=f_{\alpha+}(l, t)-f_{\alpha+}^{e q}(l, t), \\
f_{\alpha-}^{n e q}(I, t)=f_{\alpha-}(h, t)-f_{\alpha-}^{e q}(h, t) .
\end{gathered}
$$

Therefore, the general process of the present multiscale LB simulation starts from initialization to get all the necessary information, e.g., the velocity field by utilizing either the lower-order or higher-order model. The next step is to decompose the computational domain and determine the coupling interface by choosing an appropriate switching criterion. The final step is to implement the multiscale computation with lower-order models for continuum or near-continuum regime, and higher-order models for more rarefied regimes. Two models with different discrete velocity sets are coupled on the interface as described above. The second and third steps are repeated until the converged solutions are obtained.

The determination of interface, i.e., choosing an appropriate switching criterion (also called 'breakdown parameters'), is important to any coupling/hybrid strategy. Several parameters have been proposed in literature, e.g., the local Knudsen number based on the local spatial gradients of hydrodynamic variables $K n_{L}=\frac{\lambda}{\phi}\left|\frac{d \phi}{d x}\right|$ ( $\phi$ is the interested flow quantity, typically density, temperature or pressure) [49], the 'B' parameter $\left(B=\max \left\{\left|\tau_{i j}\right|,\left|q_{i}\right|\right\}\right.$

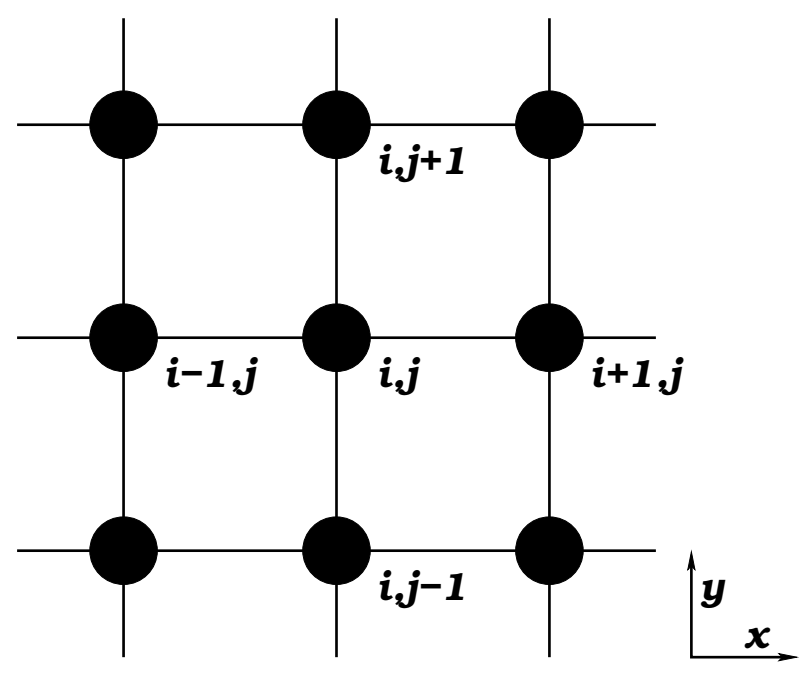

FIG. 2. Schematic diagram of square lattices.

where $\tau_{i j}$ is stress and $q_{i}$ is the heat flux[50]). These parameters are defined by macroscopic variables, and they can be used in the present LB method. However, different parameters may give significantly different values. Defining an appropriate switching criterion remains an interesting problem in itself $[3,14]$. Therefore, we do not intend to investigate the switching criterion in detail here. In the next section, we will focus on numerical test of the present multiscale LB method.

\section{NUMERICAL SIMULATIONS AND DISCUSSION}

\section{A. Numerical scheme}

To solve Eq.(16), various numerical schemes can be used. For instance, if the first-order upwind finite difference scheme is chosen, one can obtain the standard form of LB model, i.e., the stream-collision mechanism. However, for some high-order LB models, the discrete velocity points do not coincide with the lattice points. Therefore, one may choose a numerical scheme to break the tie between the time step and the lattice spacing used in the standard LB simulation[51]. As some discontinuities may occur at wall surface in the following simulations, we will employ the forward Euler time-marching method and the 2nd TVD scheme for space discretization (see Fig.2) for Eq.(16)[30, 52-54]. According to the characteristics of problems, one can also choose any other appropriate numerical method to solve Eq.(16).

Let $f_{\alpha, i}^{n, j}$ denote the distribution function value $f_{\alpha}$ at the $n$th time step in the node $\left(x_{i}, y_{j}\right)$ (see Fig.2), the 


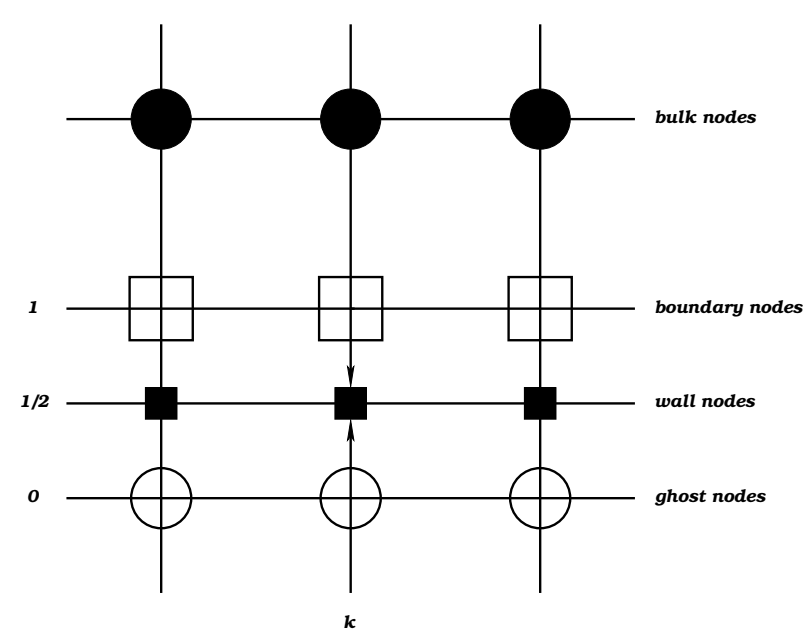

FIG. 3. Schematic illustration of wall boundary treatment.

scheme can be written as

$$
\begin{aligned}
f_{\alpha, i}^{n+1, j} & =f_{\alpha, i}^{n, j}-\frac{\xi_{\alpha x} \delta_{t}}{\delta_{x}}\left[\mathcal{F}_{\alpha, i+1 / 2}^{n, j}-\mathcal{F}_{\alpha, i-1 / 2}^{n, j}\right] \\
& -\frac{\xi_{\alpha y} \delta_{t}}{\delta_{y}}\left[\mathcal{F}_{\alpha, i}^{n, j+1 / 2}-\mathcal{F}_{\alpha, i}^{n, j-1 / 2}\right] \\
& +\frac{\delta_{t}}{K n}\left(f_{\alpha, i}^{e q, n, j}-f_{\alpha, i}^{n, j}\right)+g_{\alpha} \delta_{t},
\end{aligned}
$$

where $\delta_{x}$ and $\delta_{y}$ are the uniform grid spacing, and $\delta_{t}$ is the time step, $\xi_{\alpha x}$ and $\xi_{\alpha y}$ denote the phase velocity component at the $x$ and $y$ coordinates. The outgoing and incoming fluxes in the node $(i, j)$ (see Fig.2) are

$$
\begin{gathered}
\mathcal{F}_{\alpha, i+1 / 2}^{n, j}=f_{\alpha, i}^{n, j}+\frac{1}{2}\left(1-\frac{\xi_{\alpha x} \delta_{t}}{\delta_{x}}\right)\left[f_{\alpha, i+1}^{n, j}-f_{\alpha, i}^{n, j}\right] \Psi\left(\Theta_{\alpha, i}^{n}\right), \\
\mathcal{F}_{\alpha, i-1 / 2}^{n, j}=\mathcal{F}_{\alpha,(i-1)+1 / 2}^{n, j}, \\
\mathcal{F}_{\alpha, i}^{n, j+1 / 2}=f_{\alpha, i}^{n, j}+\frac{1}{2}\left(1-\frac{\xi_{\alpha y} \delta_{t}}{\delta_{y}}\right)\left[f_{\alpha, i}^{n, j+1}-f_{\alpha, i}^{n, j}\right] \Psi\left(\Theta_{\alpha}^{n, j}\right), \\
\mathcal{F}_{\alpha, i}^{n, j-1 / 2}=\mathcal{F}_{\alpha, i}^{n,(j-1)+1 / 2},
\end{gathered}
$$

where

$$
\begin{gathered}
\Theta_{\alpha, i}^{n}=\frac{f_{\alpha, i}^{n, j}-f_{\alpha, i-1}^{n, j}}{f_{\alpha, i+1}^{n, j}-f_{\alpha, i}^{n, j}}, \\
\Theta_{\alpha}^{n, j}=\frac{f_{\alpha, i}^{n, j}-f_{\alpha, i}^{n, j-1}}{f_{\alpha, i}^{n, j+1}-f_{\alpha, i}^{n, j}},
\end{gathered}
$$

and the minmod flux limiter is

$$
\Psi(\Theta)=\max [0, \min (1, \Theta)] .
$$

\section{B. Diffuse reflection boundary conditions}

Boundary treatment is of importance to correctly capture non-equilibrium effects, e.g. flow characteristics in

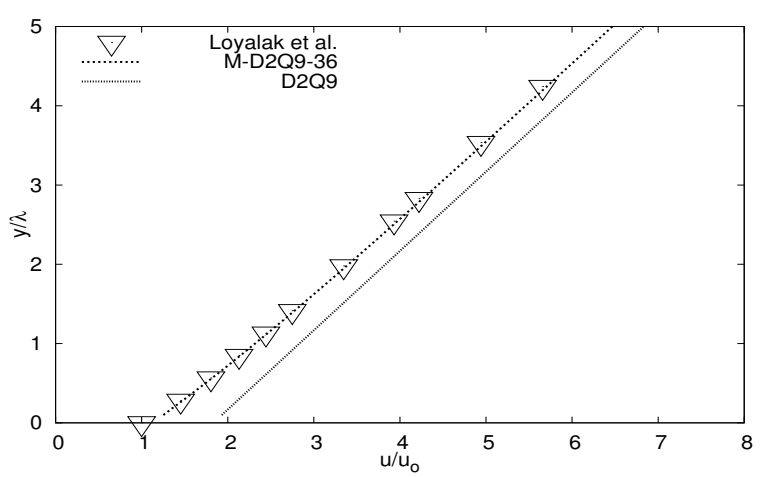

FIG. 4. The velocity profile of Kramers' problem. The symbols are the data from Loyalka et al. [58]. Here, the velocity is normalized by the reference velocity $u_{o}=-\sigma_{x y} \lambda / \mu$. The space quantity is normalized by the mean free path. The data in Ref.[58] were presented with the mean free path difined by $\mu \sqrt{2 R T} / p$. They are converted to be consistent with the present definition.

the Knudsen layer. The simple diffuse reflection model, which was developed by Maxwell in 1879 [55], has been proved to be sufficiently accurate for flows over a broad range of Knudsen numbers. The LB version of the Maxwellian model has also been developed [56]. Its specific numerical implementation on LB simulations has been discussed in Refs.[52, 57]. In this work, the Version 1 of boundary conditions in Ref.[52] will be employed.

For convenience, we assume

$$
S \approx w_{\alpha}\left\{1+\boldsymbol{\xi}_{\alpha} \cdot \boldsymbol{u}+\frac{1}{2}\left[\left(\boldsymbol{\xi}_{\alpha} \cdot \boldsymbol{u}\right)^{2}-u^{2}\right]\right\},
$$

i.e., $f_{\alpha}^{e q}=\rho S$. As the discretization is conducted along a Cartesian coordinate system (see Fig.3), the treatment of wall boundary can be described as

$$
\begin{gathered}
f_{\alpha, k}^{0}=\rho_{W, k} S\left(\boldsymbol{u}_{W, k}\right) \quad \boldsymbol{\xi}_{\alpha} \cdot \boldsymbol{n}>0 \\
\rho_{W, k}=\frac{\sum_{\left(\boldsymbol{\xi}_{\alpha} \cdot \boldsymbol{n}\right)<0}\left|\boldsymbol{\xi}_{\alpha} \cdot \boldsymbol{n}\right| f_{\alpha, k}^{1}}{\sum_{\left(\boldsymbol{\xi}_{\alpha} \cdot \boldsymbol{n}\right)>0}\left|\boldsymbol{\xi}_{\alpha} \cdot \boldsymbol{n}\right| S\left(\boldsymbol{u}_{W, k}\right)},
\end{gathered}
$$

where the subscript $W$ denotes the computational nodes at the wall, $\rho_{W, k}$ denotes the density on the wall nodes $k$ (see Fig.3), $\boldsymbol{u}_{W, k}$, the velocity, $\boldsymbol{n}$, the unity normal vector to the wall. Here, the distribution function in the ghost nodes are assumed to be identical to those on the corresponding wall nodes.

\section{Kramers' problem}

The classic Kramers' problem is often used to assess model capability in capturing the flow characteristics in the Knudsen layer (up to a few mean free paths away 
from the wall). In this problem, a gas fills the half-space $(y>0)$ bounded by a plate at $y=0$. A constant shear rate is applied along the plate at $y \rightarrow \infty$. With this special setup, one can investigate the nonlinear Knudsen layer in detail. To correctly predict this Knudsen layer, a kinetic method is required. However, for the flow region far from the plate wall, a continuum method is sufficient. Therefore, the problem is appropriate to test the coupling approach described in Sec. II B.

In the simulations, the plate is fixed at $y=0$ and a constant shear rate is applied at $y=200 \lambda$ ( $\lambda$ denotes the mean free path). The Maxwellian diffuse reflection boundary condition is employed for the fixed wall. The D2Q36 LB model is used for the region near the plate (up to $10 \lambda$ from the wall) and the D2Q9 model for the other region[59]. The results in Fig. 4 show that the nonlinear velocity profile is captured well by the multiscale LB method. It indicates that the coupling process can effectively exchange information bi-directionally.

\section{Steady Couette flow}

With a simple geometrical configure, Couette flow represents many realistic shear dominant applications, e.g. reader heads of a hard-disc driver, micro turbines and gas bearings. Moreover, Couette flow is a theoretically well defined problem. Therefore, it is generally used as a benchmark problem. Particularly, its geometry is so simple that the coupling interface can be determined easily, i.e., the flow regimes near the wall are highly rarefied and the discontinuities occur at the wall. So we can use higher-order LB models in the near-wall regions and lower-order models in the middle.

In the following simulations, the lower-order LB model will be employed for $70 \%$ of the computational region in the middle and the higher-order model for the other regions adjacent to the walls. The upper and lower plates are set to be moving oppositely with the same velocity magnitude, and the diffuse boundary condition is used for gas/wall interactions.

In Fig.5, it is clear that the D2Q9 model is unable to describe the Knudsen layer, which was also reported previously [29-31], while the M-D2Q9-36 model can obtain satisfactory results with the global Knudsen number up to 0.5. When the global Knudsen number is larger than 0.5, the multiscale method starts to deviate more from the linearized BGK (LBGK) results. This is not surprising since the Knudsen layers overlap and rarefaction effect becomes important for the whole flow domain. Note, a typical Navier-Stokes and DSMC hybrid model usually become problematic when the Knudsen number is over 0.1, e.g., see Fig.4 in Ref.[5]. To some extent, this indicates the advantage of coupling the kinetic-based LB models.

As has been shown[29-31, 33], various higher-order LB models can satisfy different requirement on model accuracy in terms of capturing high-order rarefaction effects.
Therefore, it is possible to choose LB models with appropriate discrete velocity sets according to the requirements of model accuracy and computational cost. For instance, although the D2Q36 model is used for the regions near the wall in the above simulations, the D2Q16 model may also be able to perform well for Knudsen numbers up to 0.4 , see Fig.6. Therefore, there is some flexibility in choosing various-order LB models for the present multiscale method.

\section{E. Oscillatory Couette flow}

The oscillatory Couette flows can mimic flows in many microfluidic devices containing oscillating parts. Its setup consists of a stationary plate at $y=l_{0}$ and a moving plate at $y=0$ which oscillates harmonically in the lateral direction with velocity $u=u_{W} \sin (\Omega t)$. This flow can be characterized by the Stokes number

$$
\beta=\sqrt{\frac{\rho \Omega L^{2}}{\mu}},
$$

which represents the balance between the unsteady and viscous effects. Similar to the steady case, $70 \%$ of the computational domain is computed with the lower-order LB model. The results will be compared to those of the variance-reduced (VR) particle simulations and the VR method is iscussed in the Ref.[60].

Fig.7 shows that both D2Q9 and M-D2Q9-36 models are valid in the hydrodynamic regime when the Knudsen number is low $(\mathrm{Kn}=0.0178)$. When the Knudsen number increases and the flows are in the transition regime, Figs.8 and 9 show that the D2Q9 model along becomes inappropriate while the M-D2Q9-36 model still performs well. This demonstrates that the present multiscale method can work well for the flows with various degree of rarefaction. The simulation results of the M-D2Q16-36 model as presented in Fig.10 further indicates the flexibility in choosing various-order LB models.

It is also interesting to investigate the computational performance of the multiscale method. So we test the computational performance of the D2Q9, D2Q36 and multiscale models. For the M-D2Q9-36, 10\% of the flow region is computed by the D2Q36 model and the rest is simulated by the D2Q9 model. The simulations are run on a four-core PC (Intel Core 2 QuadQ6600@2.4GHZ) without parallelization (i.e., only one core is utilized). The time required for each computational step is 0.114 ms for the M-D2Q9-36 model, 0.250 ms for D2Q36 model and $0.097 \mathrm{~ms}$ for D2Q9 model respectively. Therefore, the present multiscale approach can effectively reduce the computational costs for mixed-Kn flows. Similar to other hybrid methods, the performance of multiscale approach depends on how the computational domain is divided and calculated by the lower and higher order LB models. However, the LB framework can have some flexibility since various discrete velocity sets can be chosen 
7
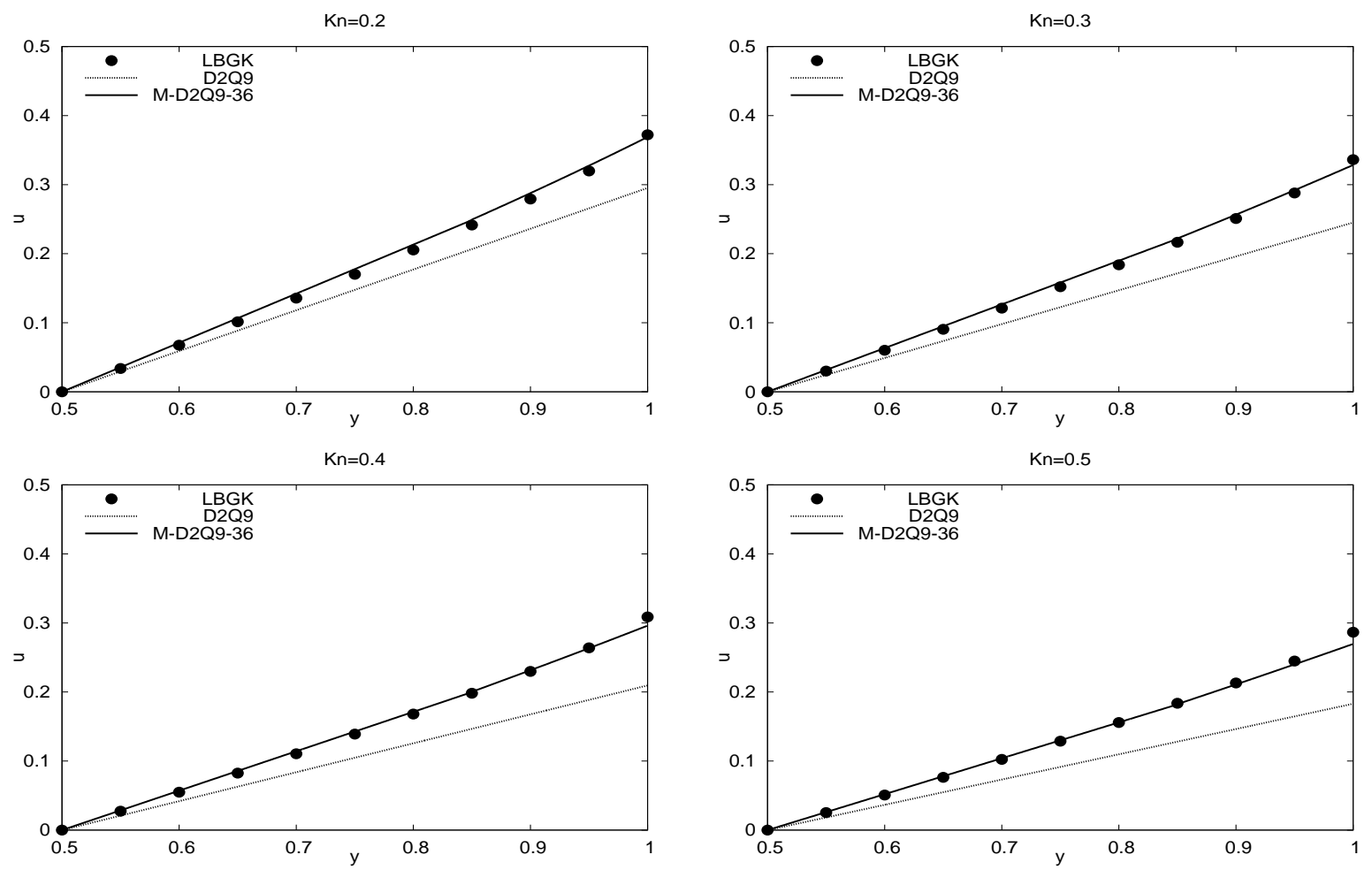

FIG. 5. Nondimensional velocity profiles for planar Couette flows where the velocity is normalized by the velocity difference between the two plates.
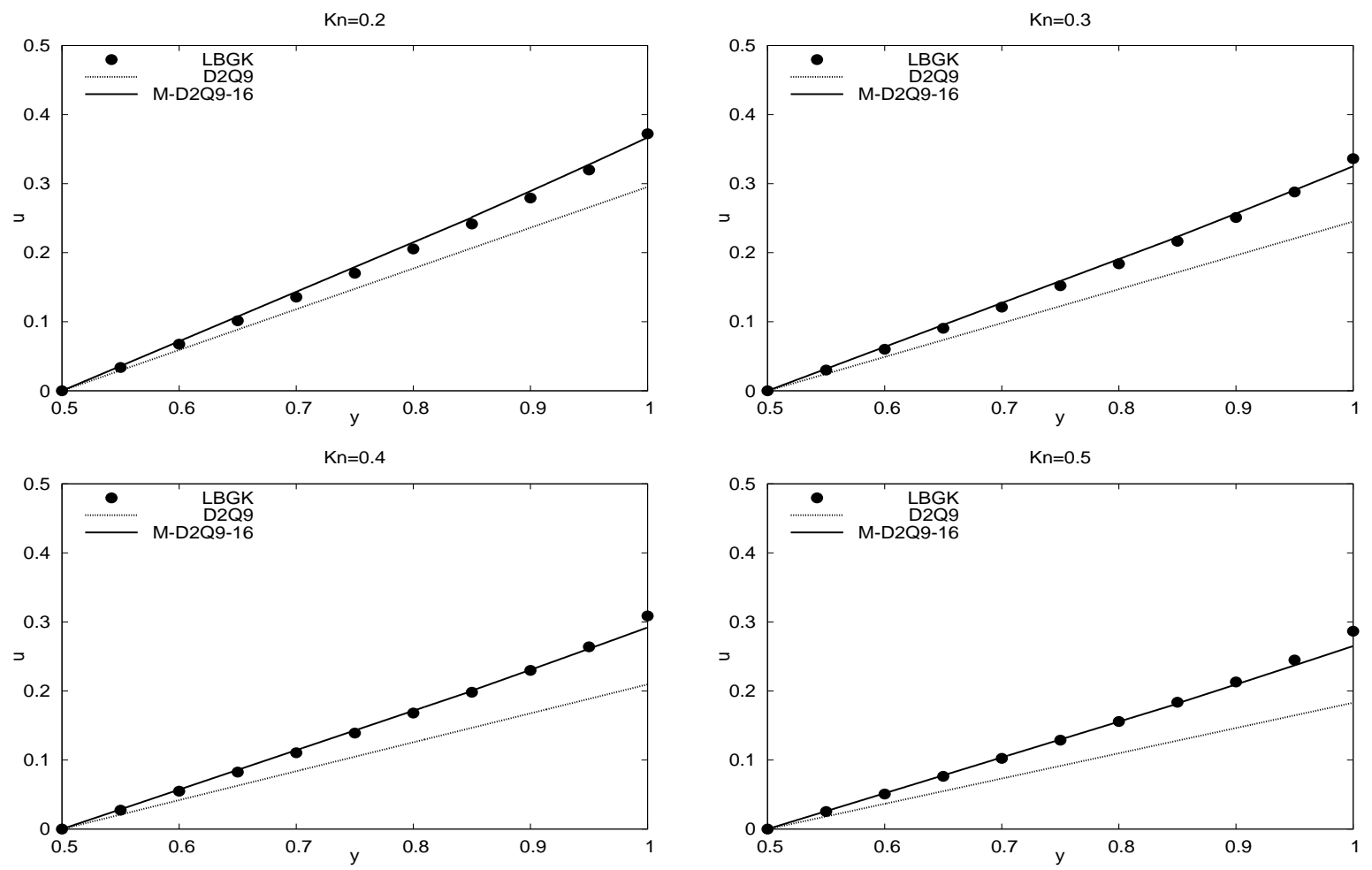

FIG. 6. Nondimensional velocity profiles for the planar Couette flows where the velocity is normalized by the velocity difference between the two plates. 

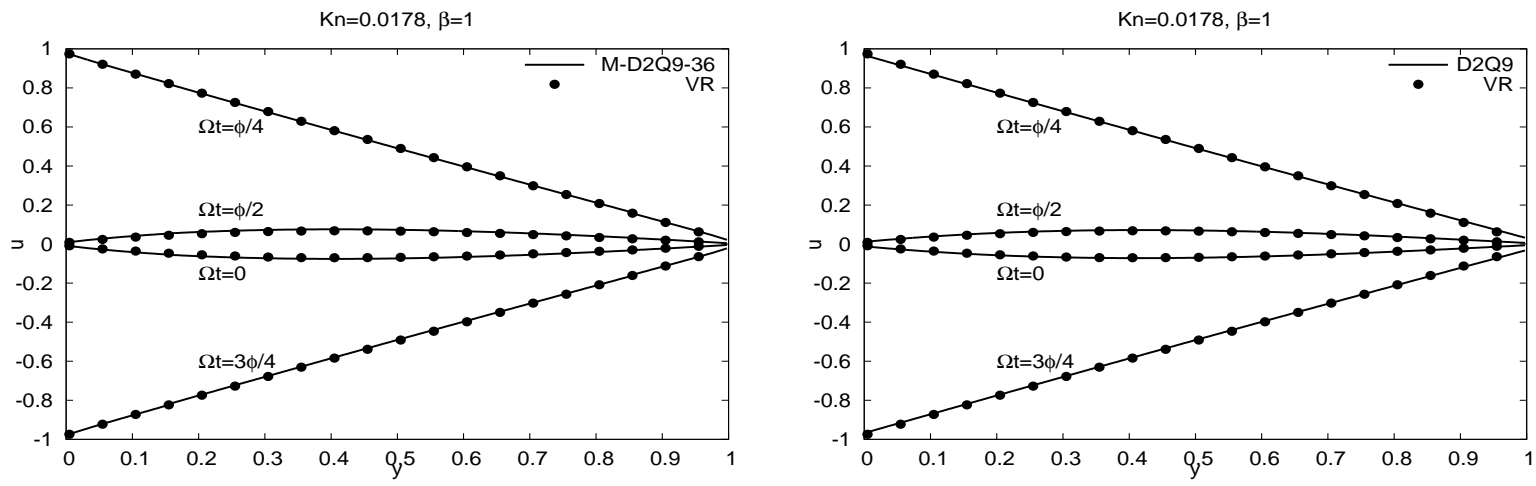

FIG. 7. Nondimensional dynamics velocity profiles for Oscillatory Couette flows where the velocity is normalized by the velocity amplitude of oscillating plate, and $\phi$ denotes the period.
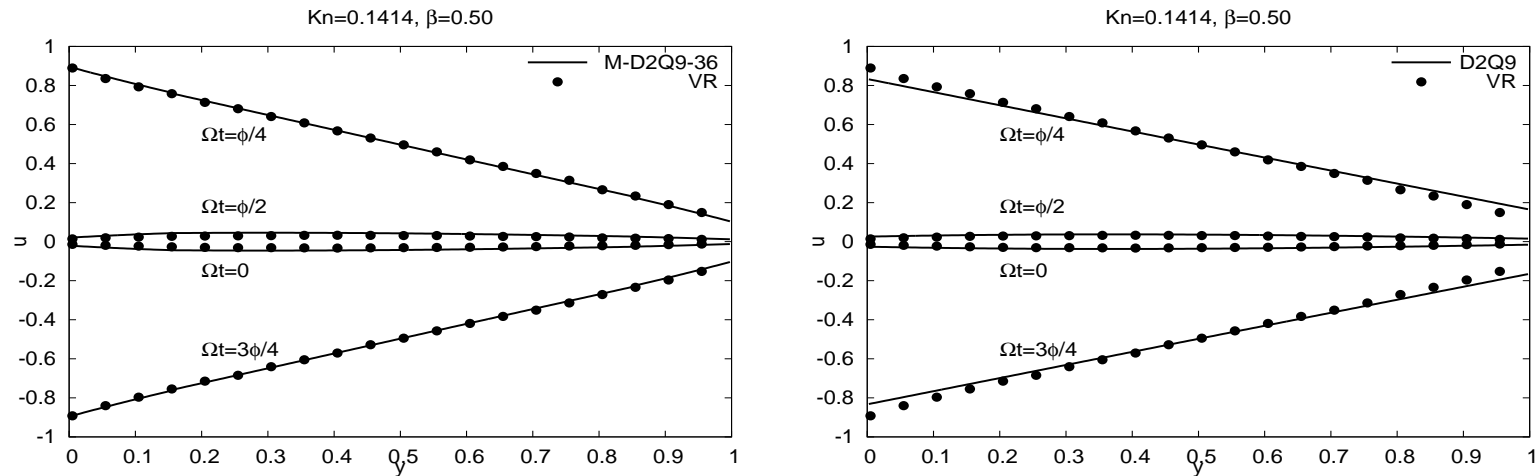

FIG. 8. Nondimensional dynamics velocity profiles for oscillatory Couette flows where the velocity is normalized by the velocity amplitude of oscillating plate, and $\phi$ denotes the period.
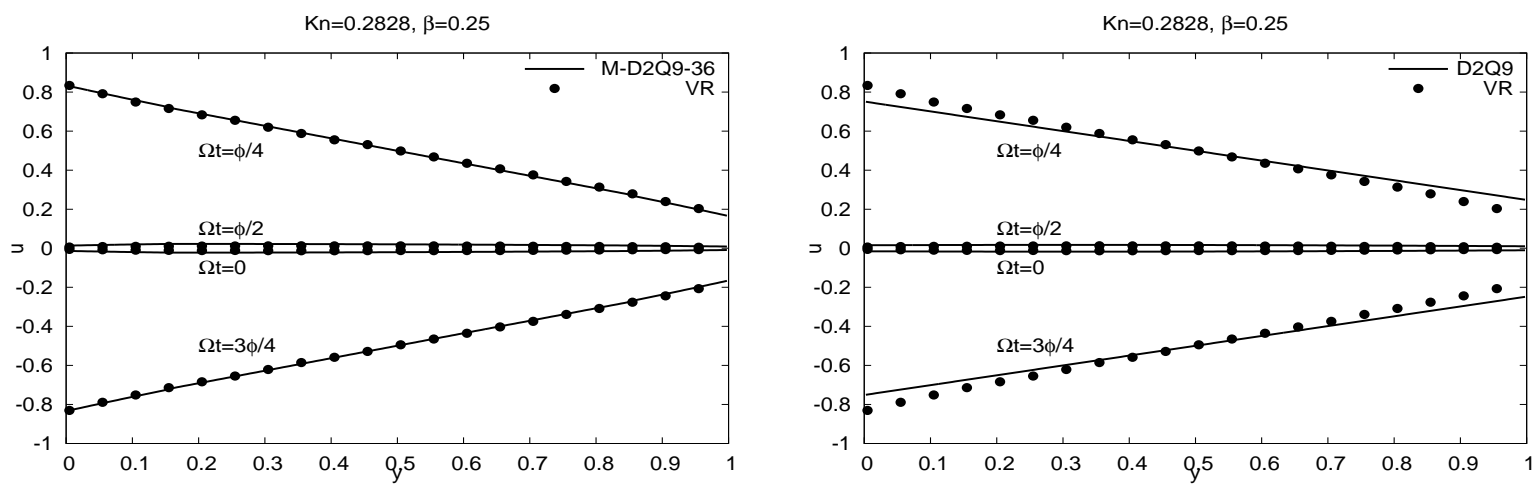

FIG. 9. Nondimensional dynamical velocity profiles for oscillatory Couette flows where the velocity is normalized by the velocity amplitude of the oscillating plate, and $\phi$ denotes the period. 


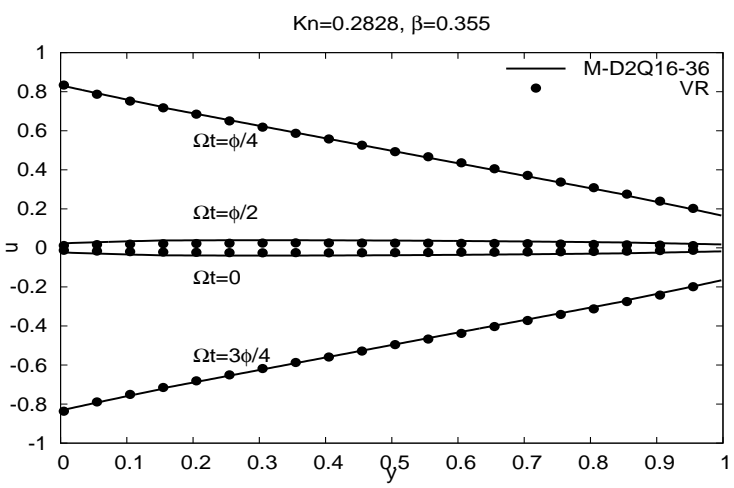

FIG. 10. Nondimensional dynamics velocity profiles for oscillatory Couette flows where the velocity is normalized by the velocity amplitude of the oscillating plate, and $\phi$ denotes the period.

to satisfy the requirement on model accuracy at the minimum computing cost. The details about how to choose appropriate LB models can be found in many references e.g., Refs.[28-31, 33, 40, 43]

As numerical stability may arise at the coupling interface, we have tested different initial flow conditions with random noises with the magnitude close to the moving wall velocity for simple Couette flow (see Fig.11). For this test case, we do not observe numerical stability problem. However, it may become an important issue for complicated flows and high-order numerical schemes. In this work, the first-order extrapolation scheme is used in the interface for exchanging non-equilibrium information. For more complicated flows, we may need to consider higher-order scheme or coupling overlapping zone to improve numerical accuracy. Finally, we have also tested the coupling scheme for a pressure-driven 2D flow in micro-channel. The Knudsen number is 0.03 at the channel outlet, and the channel length $L$ and hight $H$ are 100 and 1 respectively. The extrapolated boundary conditions are used at the inlet and outlet. And the densities at the inlet and outlet are renormalized to be $\rho_{\text {in }}=1.3$ and $\rho_{\text {out }}=1$ (see Ref.[62] for detail). For MD2Q9-36 model, the coupling interface is a cross-sectional line close to the inlet so that $8 \%$ of the channel length is simulated by the D2Q9 model. This simple case demonstrates that our multiscale model can obtain a velocity profile in good agreement with the D2Q36 model while the D2Q9 model has significant difference (see Fig.12). More effort is required to extend the proposed multiscale model for 2D and 3D complicated flows. In addition, a dynamical scheme to determine model coupling interface needs to be developed.

\section{CONCLUDING REMARKS}

A multiscale LB method utilizing low-order and highorder LB models has been developed for gas flow sim-
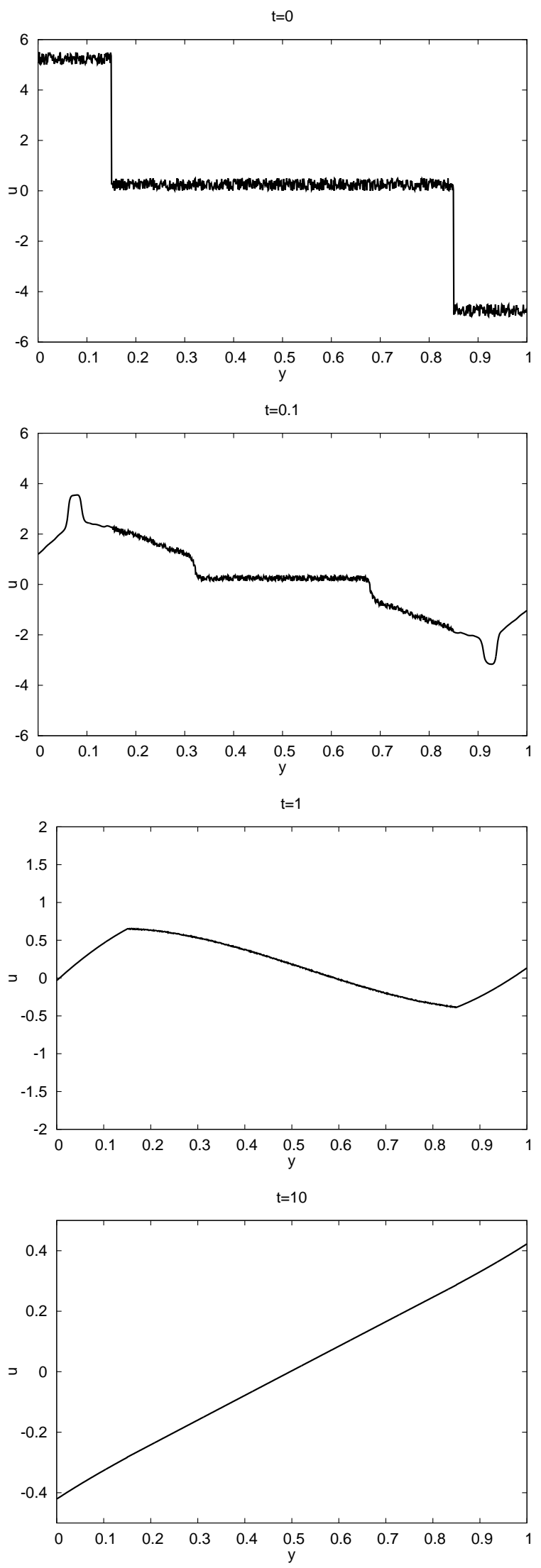

FIG. 11. The evolution of velocity profile from a random initial condition. The velocity is normalized by the velocity difference between two plates. 


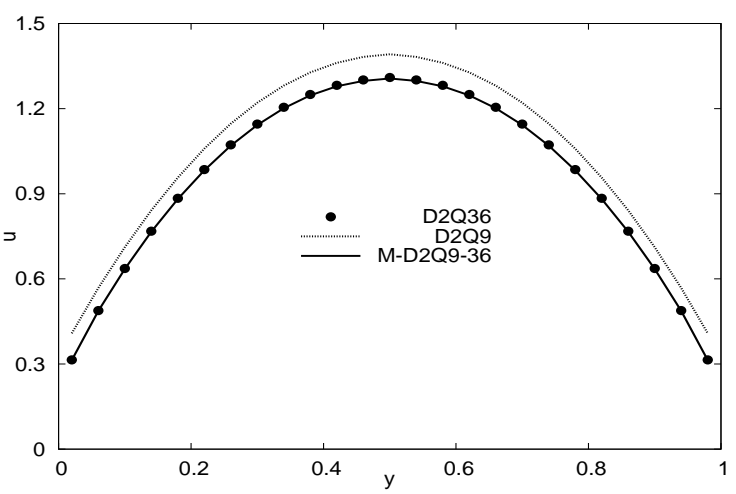

FIG. 12. The velocity profile at the outlet. The velocity is normalized by $\frac{\left(p_{\text {in }}-p_{\text {out }}\right) H^{2}}{8 L \mu}$.

ulation. As a hierarchy of LB models with various discrete velocity sets can be chosen, the multiscale method offers flexibility in designing coupling strategy to strike appropriate balance between model accuracy and computational efficiency. The present coupling process is simple by using interpolation and extrapolation processes. Therefore, the difficulty associated with kineticcontinuum hybrid models which couple two different methods becomes amenable. Furthermore, the present methodology can be extended to develop other kinetickinetic hybrid models e.g. using discrete velocity models.

\section{ACKNOWLEDGMENTS}

The authors would like to thank Nicolas Hadjiconstantinou of MIT, USA, who has performed the variancereduced particle simulations and kindly provided us the data. This work was financially supported by the Engineering and Physical Sciences Research Council U.K. under Grants No. EP/ F028865/1. The research leading to these results has received the funding from the European Community's Seventh Framework Programme FP7/2007-2013 under grant agreement ITN GASMEMS No. 215504.
$08(1)$

[45] Note1, we follow the conventional terminology for the LB models as first introduced in Ref.[61] dubbed as DnQm model i.e. $\mathrm{n}$ dimensional model with $\mathrm{m}$ discrete velocities.

[59] Note2, for convenience, the multiscale LB model will be named according to the rule M-D2Qi-j where $\mathrm{i}$ and $\mathrm{j}$ denote the discrete velocities of lower-order and higherorder model respectively.

[1] G. Karniadakis, A. Beskok, and N. Aluru, Microflows and Nanoflows: Fundamentals and Simulation (Interdisciplinary Applied Mathematics) (Springer, 2005), 1st ed.

[2] J. M. Reese, M. A., Gallis and D. A. Lockerby, Phil. Trans. R. Soc. Lond. A 361, 2967 (2003).

[3] D. A. Lockerby, J. M. Reese, and H. Struchtrup, Proc. R. Soc. A 465, 1581 (2009).

[4] S. Tiwari, A. Klar, and S. Hardt, J. Comput. Phys. 228, 7109 (2009).

[5] Q. H. Sun, I. D. Boyd, and G. V. Candler, J. Comput. Phys. 194, 256 (2004).

[6] T. E. Schwartzentruber, L. C. Scalabrin, and I. D. Boyd, J. Comput. Phys. 225, 1159 (2007).

[7] T. E. Schwartzentruber and I. D. Boyd, J. Comput. Phys. 215, 402 (2006).

[8] H. S. Wijesinghe and N. G. Hadjiconstantinou, International Journal for Multiscale Computational Engineering 2, 189 (2004).

[9] P. L. Tallec and F. Mallinger, J. Comput. Phys. 136, 51 (1997).

[10] J.-F. Bourgat, P. L. Tallec, and M. D. Tidriri, J. Comput. Phys. 127, 227 (1996).

[11] S. T. O'Connell and P. A. Thompson, Phys. Rev. E 52, R5792 (1995).

[12] J. M. Burt and I. D. Boyd, J. Comput. Phys. 228, 460 (2009).
[13] N. G. Hadjiconstantinou, Bull. Pol. Ac.: Tech 53, 335 (2005).

[14] W. L. Wang and I. D. Boyd, Phys. Fluids 15, 91 (2003).

[15] K Xu and J. C. Huang, J. Comput. Phys. 229, 7747 (2010).

[16] Y. H Qian, S. Succi, and S. Orszag, Annu. Rev. Comput. Phys. III, 195 (1995).

[17] S. Y. Chen and G. D. Doolen, Annu. Rev. Fluid Mech. 30, 329 (1998).

[18] R. Benzi, S. Succi, and M. Vergassola, Phys. Rep. 222, 145 (1992).

[19] C. K. Aidun and J. R. Clausen, Annu. Rev. Fluid Mech. 42, 439 (2010).

[20] S. Succi, I. V. Karlin, and H. D. Chen, Rev. Mod. Phys. 74, 1203 (2002).

[21] I. V. Karlin and S. Ansumali, Phys. Rev. E 76, 025701 (2007)

[22] Y. H. Zhang, R. S. Qin, and D. R. Emerson, Phys. Rev. E 71, 047702 (2005).

[23] F. Toschi and S. Succi, Europhys. Lett. 69, 549 (2005).

[24] M. Sbragaglia and S. Succi, Phys. Fluids 17, 093602 (2005).

[25] M. Sbragaglia and S. Succi, Europhys. Lett. 73, 370 (2006).

[26] G. H. Tang, Y. H. Zhang, and D. R. Emerson, Phys. Rev. E 77, 046701 (2008).

[27] Y. H. Zhang, X. J. Gu, R. W. Barber, and D. R. Emerson, Phys. Rev. E 74, 046704 (2006).

[28] X. W. Shan, X. F. Yuan, and H. D. Chen, J. Fluid Mech. 550, 413 (2006).

[29] S. Ansumali, I. V. Karlin, S. Arcidiacono, A. Abbas, and N. I. Prasianakis, Phys. Rev. Lett. 98, 124502 (2007).

[30] S. H. Kim, H. Pitsch, and I. D. Boyd, J. Comput. Phys. 227, 8655 (2008). 
[31] W. P. Yudistiawan, S. Ansumali, and I. V. Karlin, Phys. Rev. E 78, 016705 (2008).

[32] Z. L. Guo, T. S. Zhao, and Y. Shi, J. Appl. Phys. 99, 074903 (2006)

[33] J. P. Meng and Y. H. Zhang, J. Comput. Phys. 230, 835 (2011).

[34] X. Y. He and L. S. Luo, Phys. Rev. E 55, R6333 (1997).

[35] X. Y. He and L. S. Luo, Phys. Rev. E 56, 6811 (1997).

[36] X. W. Shan and X. Y. He, Phys. Rev. Lett. 80, 65 (1998).

[37] N. S. Martys, X. W. Shan, and H. D. Chen, Phys. Rev. E 58, 6855 (1998).

[38] S. Ansumali, I. V. Karlin, and H. C. Öttinger, Europhys. Lett. 63, 798 (2003).

[39] S. S. Chikatamarla and I. V. Karlin, Phys. Rev. Lett. 97, 190601(2006).

[40] S. S. Chikatamarla and I. V. Karlin, Phys. Rev. E 79, 046701 (2009)

[41] I. V. Karlin, A. Ferrante, and H. C. Öttinger, Europhys. Lett. 47, 182 (1999).

[42] S. Ansumali and I. V. Karlin, Phys. Rev. E 62, 7999 (2000).

[43] X. W Shan, Phys. Rev. E 81, 036702 (2010).

[44] H. Grad, Commun. Pure Appl. Math. 2, 331 (1949).

[45] We follow the conventional terminology for the LB models as first introduced in Ref.[61] dubbed as DnQm model i.e. $\mathrm{n}$ dimensional model with $\mathrm{m}$ discrete velocities.

[46] Z. L. Guo, C. G. Zheng, and B. C. Shi, Phys. Fluids 14, 2007 (2002).

[47] Z. L. Guo, C. G. Zheng, and B. C. Shi, Chin. Phys. 11, $366(2002)$.
[48] S. Y. Chen, D. Martínez, and R. W. Mei, Phys. Fluids 8, 2527 (1996).

[49] I. D. Boyd, G. Chen, and G. V. Candler, Phys. Fluids 7, 210 (1995).

[50] A. L. Garcia and B. J. Alder, J. Comput. Phys. 140, 66 (1998).

[51] M. Sbragaglia and K. Sugiyama, Phys. Rev. E 82, 046709 (2010).

[52] V. Sofonea, J. Comput. Phys. 228, 6107 (2009).

[53] V. Sofonea, A. Lamura, G. Gonnella, and A. Cristea, Phys. Rev. E 70, 046702 (2004).

[54] E. F. TORO, Riemann solvers and numerical methods for fluid dynamics - A practical introduction (Springer, Berlin, 1999).

[55] J. C. Maxwell, Phil. Trans. R. Soc. Lond. 170, 231 (1879).

[56] S. Ansumali and I. V. Karlin, Phys. Rev. E 66, 026311 (2002).

[57] V. Sofonea and R. F. Sekerka, J. Comput. Phys. 207, 639 (2005).

[58] S. K. Loyalka, N. Petrellis, and T. S. Storvick, Phys. Fluids 18, 1094 (1975).

[59] For convenience, the multiscale LB model will be named according to the rule M-D2Qi-j where $\mathrm{i}$ and $\mathrm{j}$ denote the discrete velocity number of lower-order and higher-order model respectively.

[60] G. A. Radtke and N. G. Hadjiconstantinou, Phys. Rev. E 79, 056711 (2009).

[61] Y. H. Qian, D. D'Humières, and P. Lallemand, Europhys. Lett. 17, 479 (1992).

[62] F. Verhaeghe, L .S. Luo, and B. Blanpain, J. Computer. Phys. 228, 147-157 (2009) 